\title{
APPROXIMATION PROPERTIES FOR ORDERINGS ON *-FIELDS
}

\author{
THOMAS C. CRAVEN
}

\begin{abstract}
The goal of this paper is to extend the main theorems on approximation properties of the topological space of orderings from formally real fields to skew fields with an involution *. To accomplish this, the concept of *-semiordering is developed and new theorems are obtained for lifting *orderings from the residue class field of a real valuation.
\end{abstract}

1. Introduction and notation. Let $\left(D,{ }^{*}\right)$ be a ${ }^{*}$-field; that is, a skew field $D$ with an involution ${ }^{*}$ (an anti-automorphism of order 2). The concept of an ordering for $\left(D,{ }^{*}\right)$ was first suggested by Reinhold Baer; a Baer ordering is a subset $P$ of the symmetric elements $S(D)=\left\{d \in D \mid d^{*}=d\right\}$ satisfying $P+P \subseteq P ; 1 \in P$; $d P d^{*} \subseteq P$ for any $d \in D^{\times}$, the nonzero elements of $D ; P \cup-P=S(D)^{\times}$; and $P \cap-P=\varnothing$. Due to a dearth of examples and the weakness of this definition, there are many unanswered questions regarding Baer orderings. In this paper, our main interest will be in a stronger notion corresponding to the usual definition of ordering on a commutative field. We need some notion of closure under multiplication. The first proposal was that of a "strong ordering" by Holland [H2]. A very closely related alternative is a "Jordan ordering" proposed by Idris in [I] . A theory for lifting these, as well as the more general Baer orderings, is developed in $[\mathbf{C r}]$. In this paper we take the liberty of changing the terminology a little in order to create a more coherent connection with the commutative theory.

DEFINITION 1.1 ("JORDAN ORDERING" OF $[\mathbf{I}]$ ). A *-ordering of $\left(D,{ }^{*}\right)$ is a Baer ordering $P$ which also satisfies the condition that $x y+y x \in P$ whenever $x, y \in P$.

DEFINITION 1.2 ("STRONG ORDERING" OF [H2]). An extended *-ordering of $\left(D,{ }^{*}\right)$ is a *-closed subset $P$ of $D$ satisfying $P+P \subseteq P ; 1 \in P ; d P d^{*} \subseteq P$ for any $d \in D^{\times} ; P \cup-P \supseteq S(D)^{\times} ; P \cap-P=\varnothing$; and $P \cdot P \subseteq P$.

It is shown in $[\mathbf{I}]$ that if $P$ is an extended *-ordering, then $P \cap S(D)$ is a *-ordering, and any *-ordering $P$ is contained in a unique maximal extended *-ordering which we shall denote by $P^{e}$. We shall usually find it more convenient to work with *orderings. Note that either of these concepts gives the usual notion of ordering on a commutative field with ${ }^{*}=$ identity. Just as in the commutative case, the set $X_{D}$ of all *-orderings of $D$ can be topologized (see $\S 4$ for details). One can then ask when subsets of $X_{D}$ can be separated by an element $d \in D$ (i.e. $d$ is positive on one subset, negative on the other). This question was first raised in [KRW]

Received by the editors April 7, 1987 and, in revised form, October 17, 1987.

1980 Mathematics Subject ('lassification (1985 Revision). Primary 12E15, 16A28; Secondary 12D15, 16A39.

Key words and phrases. Bacr ordering, "-valuation, SAP. 
and $[\mathbf{E L}]$. Since then, many authors have written about the "strong approximation property" (SAP), which states that any two closed sets can be so separated. The main purpose of this paper is to generalize to ${ }^{*}$-fields the characterizations given in [P1, Theorem 9.1] (see Theorem 4.1). The most important conditions equivalent to SAP are

(1) the valuation theoretic characterization: for every real valuation $v$, we have $\left|S\left(\Gamma_{v}\right) / 2 \Gamma_{v}\right| \leq 2$, and if equal to 2 , then $\left|X_{D_{v}}\right|=1$; and

(2) every ${ }^{*}$-semiordering is a ${ }^{*}$-ordering.

Superficially, these characterizations appear almost the same as their commutative (with ${ }^{*}=$ identity) counterparts. However, the terms "real valuation" and "*-semiordering" must be very carefully defined to make it work (see $\S 2$ ). In particular, the concept of Baer ordering is too weak a generalization of the commutative notion of semiordering. Most of the work of this paper is new for ${ }^{*}$-orderings of commutative fields (with ${ }^{*} \neq$ identity) as well as for skew fields. Some work on the commutative case can be found in $[\mathbf{K}]$, though the emphasis there is on commutative rings.

$\S 3$ of this paper extends the lifting theorems of $[\mathbf{C r}]$ to ${ }^{*}$-semiorderings and develops a new lifting theorem for *-orderings with apparently weaker hypotheses than those found in $[\mathbf{C r}]$.

For any subset $C$ of $D$, we shall write $S(C)$ for the set of symmetric elements in $C$, i.e. $S(C)=\left\{c \in C \mid c^{*}=c\right\}$. We write $C^{\times}$for the set of nonzero elements in $C$, and we write $\Pi S(D)$ for the set of all nonzero products of symmetric elements of $D$.

We shall write $[a, b]$ for the commutator $a b a^{-1} b^{-1}$ and $[A, B]$ for the subgroup of the multiplicative group $D^{\times}$generated by $\{[a, b] \mid a \in A, b \in B\}$, where $A, B \subseteq D^{\times}$. We refer to elements of the form $d d^{*}$ as norms.

DEFINITION 1.3. We write $\Sigma=\Sigma(D)$ for the set of all sums of products of norms and elements of $\left[D^{\times}, \Pi S(D)\right]$.

The sets $\Sigma$ and $S(\Sigma)$ play the role here that sums of squares do in the ArtinSchreier theory of orderings. For example, Holland [H2] has shown that $D$ has a *-ordering iff $0 \notin \Sigma(D)$ and that the intersection of all *-orderings of $D$ is $S(\Sigma)$.

Let $v$ be a valuation on $D$ in the sense of $[\mathbf{S}]$. We denote the valuation ring, its maximal ideal, its group of units, residue class (skew) field and value group by $A_{v}, \mathfrak{m}_{v}, U_{v}, D_{v}$ and $\Gamma_{v}$, respectively. For an element $d \in D$ with $v(d)=0$, we shall write $\bar{d}$ for the image of $d$ in $D_{v}$. Our valuations will generally be required to be ${ }^{*}$-valuations as defined in $[\mathbf{H 1}]$, which is to say $v(d)=v\left(d^{*}\right)$ for $d \in D^{\times}$. It is easily seen that this forces $\Gamma_{v}$ to be abelian, so we shall write our valuations additively. We write $S\left(\Gamma_{v}\right)$ for the subset $\left\{\gamma \in \Gamma_{v} \mid v(d)=\gamma\right.$ for some $\left.d \in S(D)\right\}$. $\mathrm{A}^{*}$-valuation is said to be compatible with an ordering $P$ (of any type) if whenever $0<a \leq b$ with respect to $P$, then $v(a) \geq v(b)$.

We state the following result from $[\mathbf{C r}]$ since it will be used so often here.

LEMMA 1.4 (CF. [Cr, LEMMA 2.6 AND REMARKS FOLLOWING]). (a) Let $v$ be $a^{*}$-valuation on $\left(D,{ }^{*}\right)$ and let $d \in \Pi S(D)$. Then $v\left(d+d^{*}\right)=v(d)$ if either of the following conditions hold:

(i) $v\left(1+a b a^{-1 *} b^{-1}\right) \leq 0$ for any $a \in \Pi S(D), b \in S(D)^{\times}$.

(ii) $v$ is compatible with $a^{*}$-ordering.

(b) When the conclusion of (a) holds, the set $S\left(\Gamma_{v}\right)$ is a subgroup of $\Gamma_{v}$. 
Given a Baer ordering $P$, there is one particular "valuation" in which we shall be particularly interested. This is the one obtained by looking at the archimedean classes in $D$ determined by $P$. Let $A=\left\{d \in D \mid 0 \leq d d^{*}<n\right.$ for some integer $\left.n\right\}$. Holland [H1, Theorem 4.3] has shown that $A$ is a *-closed local subring of $D$ with residue (skew) field isomorphic to a subfield of $\mathbf{R}, \mathbf{C}$ or $\mathbf{H}$ (the real quaternions) with $P$ inducing a Baer ordering contained in $\mathbf{R}^{+}$on the residue field. Since the residue field is a subfield of $\mathbf{H}$, we have $S\left(D_{v}\right) \subseteq Z\left(D_{v}\right)$, the center of $D_{v}$. By [P1, Theorem 1.20], this induced Baer ordering, being archimedean, is actually closed under multiplication, hence is a *-ordering of the residue field. A "valuation" can be defined corresponding to $A$ which Holland calls the order valuation. It is, generally, a valuation only in a more general sense than that of $[\mathbf{S}]$. However it is a ${ }^{*}$-valuation if $P$ is a ${ }^{*}$-ordering $[\mathbf{H 2}, \mathbf{I}]$. Much of $\S 2$ of this paper is devoted to showing that $v$ is a ${ }^{*}$-valuation under the less restrictive hypothesis that $P$ is a ${ }^{*}$-semiordering as defined in $\S 2$.

2.*-semiorderings. One has some choice in how to extend the definition of (formally) real valuation to skew fields with involution. For our purposes, the following definition will work best.

DEFinition 2.1. A *-valuation $v$ on $\left(D,{ }^{*}\right)$ is real if $0 \notin \Sigma\left(D_{v}\right)$ and $\overline{\left[D^{\times}, S(D)^{\times}\right]}$ $\subseteq \Sigma\left(D_{v}\right)$.

If $\Gamma$ is the value group of a real valuation $v$, then $S(\Gamma)$ is a group by Lemma 1.4. If $D$ is commutative, then the condition of reality for $v$ reduces to $0 \notin \Sigma\left(D_{v}\right)$, which is equivalent to $D_{v}$ being formally real. (See $[\mathbf{K}]$ for ${ }^{*} \neq$ id and for generalizations to commutative rings.) The main motivation for the commutator condition on a real *-valuation is the following proposition. Its proof requires the main lifting theorem of the next section. We state and prove it out of order since it motivates the definition and will not be used henceforth.

PROPOSITION 2.2. Let $v$ be $a^{*}$-valuation.

(a) If $v$ is real, then $\Sigma(D) \cap U_{v}$ maps onto $\Sigma\left(D_{v}\right)$.

(b) The valuation $v$ is real iff $D_{v}$ has $a^{*}$-ordering and every ${ }^{*}$-ordering of $D_{v}$ has a lifting to $a^{*}$-ordering of $D$ (compatible with $v$ ).

ProOF. Assume that $v$ is real. Since $0 \notin \Sigma\left(D_{v}\right)$, the ${ }^{*}$-field $D_{v}$ has a *-ordering [H2, Theorem 3.9]. This ${ }^{*}$-ordering lifts to $D$ by Theorem 3.4 to a ${ }^{*}$-ordering $P$ compatible with $v$, and thus half of (b) is proved. Now any element $d \in \Sigma(D) \cap U_{v}$ is a sum of products of norms and commutators, so each summand lies in $P^{e}$. By $[\mathrm{Cr}$, Lemma 2.4(a)], the value of $d$ is the minimum of the values of the summands, so each summand lies in $A_{v}$. Since $v$ is real, the image in $D_{v}$ of each summand not in $\mathrm{m}_{v}$ is a product of norms in $D_{v}$ and elements of $\Sigma\left(D_{v}\right)$, hence lies in $\Sigma\left(D_{v}\right)$. It follows that $\bar{d} \in \Sigma\left(D_{v}\right)$, proving (a).

For the converse of (b), we have $0 \notin \Sigma\left(D_{v}\right)$ since $D_{v}$ has a ${ }^{*}$-ordering. The group $\left[D^{\times}, S(D)^{\times}\right]$is contained in every extended *-ordering of $D$, and hence by hypothesis $\overline{\left[D^{\times}, S(D)^{\times}\right]}$is contained in every extended * -ordering of $D_{v}$. By $[\mathbf{H 2}, \mathrm{p}$. $26]$, the intersection of these is $\Sigma\left(D_{v}\right) \cap \Pi S\left(D_{v}\right)$, so we obtain $\overline{\left[D^{\times}, S(D)^{\times}\right]} \subseteq \Sigma\left(D_{v}\right)$ as desired.

Following [H2], we shall say that the valuation $v$ collapses a subgroup $G$ of $D^{\times}$ if $v(1-d)>0$ for all $d \in G$. 
PROPOSITION 2.3. Either of the following conditions is sufficient to guarantee that a valuation $v$ on $a^{*}$-field $D$ with $0 \notin \Sigma(D)$ is real:

(a) $v$ collapses $\left[S(D)^{\times}, S(D)^{\times}\right]$and $\left[D^{\times}, D^{\times}\right] \subseteq A_{v}$.

(b) $v$ is $a^{*}$-valuation which collapses $\left[S(D)^{\times}, S(D)^{\times}\right]$.

PROOF. From Holland $[\mathbf{H 2}, 2.2]$ we have that $v$ is a ${ }^{*}$-valuation iff $\left[D^{\times}, D^{\times}\right] \subseteq$ $A_{v}$. Thus (a) and (b) are equivalent. If $v$ collapses $\left[S(D)^{\times}, S(D)^{\times}\right]$, then $v$ collapses all of $\left[D^{\times}, S(D)^{\times}\right]$to $1 \in \Sigma\left(D_{v}\right)$ by [Cr, Theorem 2.3].

DEFINITION 2.4. $\mathrm{A}^{*}$-semiordering of $\left(D,{ }^{*}\right)$ is a Baer ordering $P$ satisfying

$$
p x+x^{*} p \in P \quad \text { whenever } p \in P, x \in \Sigma(D) .
$$

The set of all *-semiorderings of $\left(D,{ }^{*}\right)$ will be denoted by $Y_{D}^{*}$.

Every *-ordering is a *-semiordering [H2, Theorem 3.4(3)]. Every Baer ordering of a commutative field is a ${ }^{*}$-semiordering since $\Sigma=S(\Sigma)$ is the set of sums of norms and for $x=t t^{*}$, the element $p x+x^{*} p=2 t p t^{*}$ lies in the Baer ordering.

LEMMA 2.5. Let $P$ be $a^{*}$-semiordering and let $B=\left\{\sum p_{i} d_{i} \mid p_{i} \in P, d_{i} \in \Sigma\right\}$. Then $S(B) \subseteq P$.

Proof. Let $x \in S(B)$, say $x=\sum p_{i} d_{i}, p_{i} \in P, d_{i} \in \Sigma$. Then $x=\left(x+x^{*}\right) / 2=$ $\sum\left(p_{i} d_{i}+d_{i}^{*} p_{i}\right) / 2 \in P$.

For $\left\{s_{i}\right\} \subseteq S(D)^{\times}$, define $\Sigma\left(\left\{s_{i}\right\}\right)=\left\{\sum s_{i} x_{i}+x_{i}^{*} s_{i} \mid x_{i} \in \Sigma \cup\{0\}\right.$, not all $\left.x_{i}=0\right\}$. Note that $\Sigma\left(\left\{s_{i}\right\}\right) \subseteq S(D), \Sigma(1)=S(\Sigma)$ and $\Sigma(P)=P$ for any ${ }^{*}$-semiordering $P$.

PROPOSITION 2.6. Let $\left\{s_{i}\right\} \subseteq S(D)$ such that $0 \notin \Sigma\left(\left\{s_{i}\right\}\right)$. Then there exists $a^{*}$-semiordering $P$ containing either $\Sigma\left(\left\{s_{i}\right\}\right)$ or $\Sigma\left(\left\{-s_{i}\right\}\right)$.

Proof. Assurne $0 \in \Sigma\left(\left\{s_{i}\right\}\right)+S(\Sigma)$. Then we claim that $0 \notin \Sigma\left(\left\{-s_{i}\right\}\right)+S(\Sigma)$. Say $0=\sum s_{i} x_{i}+x_{i}^{*} s_{i}+s$ for some $x_{i} \in \Sigma \cup\{0\}, s \in S(\Sigma)$, and also $0=-\sum s_{i} y_{i}+$ $y_{i}^{*} s_{i}+t$ for some $y_{i} \in \Sigma \cup\{0\}, t \in S(\Sigma)$. Then

$$
\begin{aligned}
0 & =t s^{-1} \Sigma\left(s_{i} x_{i}+x_{i}^{*} s_{i}\right)+t s^{-1} s+\Sigma\left(s_{i} y_{i}+y_{i}^{*} s_{i}\right)-t \\
& =\Sigma\left(t s^{-1} s_{i} x_{i}+t s^{-1} x_{i}^{*} s_{i}\right)+\Sigma\left(s_{i} y_{i}+y_{i}^{*} s_{i}\right) \\
& =\sum d_{i} s_{i},
\end{aligned}
$$

where

$$
d_{i}=t s^{-1} x_{i}\left[x_{i}^{-1}, s_{i}\right]+t s^{-1} x_{i}^{*}+y_{i}\left[y_{i}^{-1}, s_{i}\right]+y_{i}^{*} \in \Sigma .
$$

Thus $0=\sum d_{i} s_{i}+s_{i} d_{i}^{*} \in \Sigma\left(\left\{s_{i}\right\}\right)$, a contradiction.

So without loss of generality we may assume that $0 \notin \Sigma\left(\left\{s_{i}\right\}\right)+S(\Sigma)$. Let $P$ be a maximal subset of $S(D)^{\times}$such that $P \supseteq \Sigma\left(\left\{s_{i}\right\}\right)+S(\Sigma), P+P \subseteq P, d P d^{*} \subseteq P$ $\left(d \in D^{\times}\right), P \cap-P=\varnothing$ and $\left(S^{*}\right)$ holds. If $P$ is not a ${ }^{*}$-semiordering, then there must be some element $s \in S(D)^{\times}$with $s \notin \pm P$. The maximality of $P$ implies that we can write

$$
0=\sum x_{i} s x_{i}^{*}+s d+d^{*} s+p, \quad \text { where } x_{i} \in D^{\times}, p \in P, d \in \Sigma .
$$

Then $s q=-p$, where

$$
q=\sum\left[s^{-1}, x_{i}\right] x_{i} x_{i}^{*}+d+\left[s^{-1}, d^{*}\right] d^{*} \in \Sigma,
$$

and so $s=-p q^{-1} \in S(D) \cap-P \cdot \Sigma \subseteq-P$ by Lemma 2.5 , a contradiction of the choice of $s$. 
LEMMA 2.7. Let $P$ be $a^{*}$-semiordering of $\left(D,{ }^{*}\right)$.

(a) If $s \in S(D)^{\times}$and $p \in P$, then $s^{-1} p s+s p s^{-1} \in P$.

(b) If $x \in \Pi S(D)$, then $x^{2}+x^{* 2} \in P$.

PROOF. For (a) we have sps $\in P$ and $s^{-2} \in S(\Sigma)$, so $s^{-1} p s+s p s^{-1}=s^{-2}(s p s)+$ $(s p s) s^{-2} \in P$. For (b), we have $x x^{*-1} \equiv 1\left(\bmod \left[S(D)^{\times}, S(D)^{\times}\right]\right)$, so $x x^{*-1}+$ $x^{-1} x^{*} \in S(\Sigma) \subseteq P$. Thus $x^{2}+x^{* 2}=x\left(x x^{*-1}+x^{-1} x^{*}\right) x^{*} \in P$.

PROPOSITION 2.8. Let $P$ be $a^{*}$-semiordering of $\left(D,{ }^{*}\right)$ compatible with $a^{*}$ valuation $v$. Then for any $d \in \Pi S(D)$, we have $v\left(d+d^{*}\right)=v(d)$.

ProOF. We have $v\left(d+d^{*}\right) \geq v(d)=v\left(d^{*}\right)$, or equivalently, $v\left(d d^{*-1}+1\right) \geq 0$. Set $c=d d^{*-1}$, and assume $v(c+1)>0$. Then also $v\left(c^{*}+1\right)>0$, hence $v\left(c+c^{*}+2\right)>0$. But $c+c^{*}=d\left(d^{*-2}+d^{-2}\right) d^{*} \in P$. Thus $0<2<c+c^{*}+2$, so by compatibility, we obtain the desired contradiction $0<v\left(c+c^{*}+2\right) \leq v(2)=0$.

THEOREM 2.9. If $a^{*}$-valuation $v$ is compatible with $a^{*}$-semiordering $P$, then $S\left(\Gamma_{v}\right)$ is a subgroup of $\Gamma_{v}$.

PROOF. Given $s_{i} \in S(D)^{\times}$with $v\left(s_{i}\right)=\gamma_{i}$, we obtain $v\left(s_{1} s_{2}^{-1}+s_{2}^{-1} s_{1}\right)=\gamma_{1}-\gamma_{2}$ by Proposition 2.8 .

At this point we wish to fix some notation for the remainder of this section. We consider a fixed *-semiordering $P$ and its order valuation $v$. We write $A$ for the *-subring of finite elements (with respect to $\mathbf{Q}$ ), $\mathfrak{m}=\left\{d \in D \mid d d^{*}<q\right.$ for all positive $q \in \mathbf{Q}\}$ for its maximal ideal of infinitesimal elements and $U$ for the group of units of $A$. Our goal is to show that $v$ is a real *-valuation. Then we will know that $A$ is a *-valuation ring and the set of values form an abelian group $\Gamma[\mathbf{H 1}, 4.6]$.

LEMMA 2.10. (a) If $s \in S(D)^{\times}, x \in \mathfrak{m}$, then $s x s^{-1} \in \mathfrak{m}$.

(b) If $x \in D^{\times}$, then $x^{-1} x^{*} \in U$.

PROOF. (a) Since $x=\left(x+x^{*}\right) / 2+\left(x-x^{*}\right) / 2$, with both terms in $\mathfrak{m}$, the first term symmetric and the second term skew, it suffices to consider these two cases. First assume $x=x^{*}$. Since $x \in \mathfrak{m}$, we have $x<q$ for all $q \in \mathbf{Q}^{+}$. But then Lemma 2.7(a) implies that $2 q-\left(s x s^{-1}+s^{-1} x s\right)=s(q-x) s^{-1}+s^{-1}(q-x) s \in P$ for all $q>0$, so we have $s x s^{-1}+s^{-1} x s \in \mathrm{m}$. Now set $d=s x s^{-1}$. Then we obtain

$$
\begin{aligned}
d d^{*}+d^{*} d & =s x s^{-2} x s+s^{-1} x s^{2} x s^{-1} \\
& =\left(s x s^{-1}+s^{-1} x s\right)^{2}-\left(s x^{2} s^{-1}+s^{-1} x^{2} s\right) \in \mathfrak{m} \cap P,
\end{aligned}
$$

and thus $d d^{*}$, and hence $d$, lie in $\mathrm{m}$. Next we assume $x=-x^{*}$. By the symmetric case, $\left(s x s^{-1}\right)^{2}=s x^{2} s^{-1} \in \mathrm{m}$, and hence $s x s^{-1} \in \mathfrak{m}$.

(b) Case 1: $x+x^{*}=0$. Then $x^{-1} x^{*}=-1 \in U$.

Case 2: $x+x^{*} \neq 0$. Then an easy computation shows

$$
\left(x+x^{*}\right) x^{-1} x^{*}\left(x+x^{*}\right)^{-1}=x^{*} x^{-1} .
$$

Now if $x^{-1} x^{*} \in \mathfrak{m}$, then (a) implies $\left(x^{-1} x^{*}\right)^{-1}=\left(x^{*} x^{-1}\right)^{*} \in \mathfrak{m}$, a contradiction. Similarly, $\left(x^{-1} x^{*}\right)^{-1}$ cannot lie in $\mathfrak{m}$, so $x^{-1} x^{*} \in U$.

LEMMA 2.12. (a) If $s \in S(D) \cap U, d \in S(D)^{\times}$, then $d s d^{-1} \equiv s(\bmod \mathrm{m})$.

(b) If $s \in S(D)^{\times}, d \in D^{\times}$, then $[s, d] \not \equiv-1(\bmod \mathrm{m})$.

(c) If $x, y \in D^{\times}$with $\left[x^{2}, y\right] \equiv 1,[x, y] \not \equiv 1$, then $[x, x y-y x] \equiv-1(\bmod m)$. 
(d) If $s \in S(D)^{\times}, x \in U$, then $[s, x] \equiv 1(\bmod \mathrm{m})$.

(e) If $x, y \in D^{\times}$, then $[x, y][x, y]^{*} \equiv 1(\bmod \mathfrak{m})$.

ProOF. (a) Without loss of generality, we may assume that $s \in P$. Since $s \in U$, there exist $q_{1}, q_{2} \in \mathbf{Q}^{+}$such that $q_{1} \leq s \leq q_{2}$. Applying Lemma 2.7(a), we obtain $q_{1} \leq\left(d s d^{-1}+d^{-1} s d\right) / 2 \leq q_{2}$. Since this holds for any rational bounds $q_{i}$ on $s$, we have $\left(d s d^{-1}+d^{-1} s d\right) \equiv 2 s(\bmod \mathfrak{m})$. Now define automorphisms $\varphi: D_{v} \rightarrow D_{v}$ by $\varphi(\bar{x})=\overline{d x d^{-1}}$, and $\psi: D_{v} \rightarrow D_{v}$ by $\psi=\left(\varphi+\varphi^{*}\right) / 2$. We have shown that $\psi(\bar{s})=\bar{s}$ for $s \in S(D)$; we must show that $\varphi(\bar{s})=\bar{s}$. Write $\varphi(\bar{s})=\bar{s}+\bar{k}, \varphi^{*}(\bar{s})=\bar{s}-\bar{k}$. Then $\bar{k}^{*}=-\bar{k}$ and we may assume that $k$ is skew as well (if $k^{*}=-k+m, m \in \mathfrak{m}$, then replace $k$ by $k-m / 2)$. Using the fact that $S\left(D_{v}\right) \subseteq Z\left(D_{v}\right)$, we obtain

$$
\begin{aligned}
\varphi\left(\overline{(s+k)(s+k)^{*}}\right) & =\varphi\left(\overline{s^{2}-k^{2}}\right)=(\bar{s}+\bar{k})^{2}-\varphi(\bar{k})^{2} \\
& =\bar{s}^{2}+2 \bar{s} \bar{k}+\bar{k}^{2}-\varphi(\bar{k})^{2}
\end{aligned}
$$

and

$$
\varphi^{*}\left(\overline{(s+k)(s+k)^{*}}\right)=\bar{s}^{2}-2 \bar{s} \bar{k}+\bar{k}^{2}-\varphi(\bar{k})^{2} .
$$

Thus $\psi\left(\overline{(s+k)(s+k)^{*}}\right)=\bar{s}^{2}+\bar{k}^{2}-\left(\varphi(\bar{k})^{2}+\varphi^{*}(\bar{k})^{2}\right) / 2$. Since $\psi$ fixes symmetrics, we also have $\psi\left(\overline{(s+k)(s+k)^{*}}\right)=\psi\left(\overline{s^{2}-k^{2}}\right)=\bar{s}^{2}-\bar{k}^{2}$. Combining these gives $\psi\left(\bar{k}^{2}\right)=2 \bar{k}^{2}$. Since we also have $\psi\left(\bar{k}^{2}\right)=\bar{k}^{2}$, we obtain $\bar{k}=0$ completing the proof of (a).

(b) Assume that $[s, d]=-1+m, m \in \mathfrak{m}$. By Lemma $2.5[s, d]+[s, d]^{*} \in P$, hence $\overline{-2+m+m^{*}}=-2 \in \bar{P}$, a contradiction.

(c) By hypothesis, $x[x, y] x^{-1}[x, y]=\left[x^{2}, y\right] \equiv 1$. Setting $z=[x, y]$ and using $x z x^{-1} \equiv z^{-1}$, a computation shows that

$$
\begin{aligned}
{[x, x y-y x] } & =\left(x z x^{-1}-1\right) z(z-1)^{-1} \\
& \equiv\left(z^{-1}-1\right) z(z-1)^{-1}=-1 .
\end{aligned}
$$

(d) Case 1: $x^{*}=-x$. Since $x^{2} \in S(D)$, part (a) implies $\left[s, x^{2}\right] \equiv 1$. If $[s, x] \not \equiv 1$, then part (c) implies $[x, s x-x s] \equiv-1$, contradicting (b).

Case 2: $x=t+k \in U$ with $t^{*}=t, k^{*}=-k$. Then $t, k \in A$; using (a) and Case 1 , we obtain $s x s^{-1}=s t s^{-1}+s k s^{-1} \equiv t+k=x$.

(e) Since $[x, y]=\left(\left(x^{*}\right)^{*}\left(x^{*}\right)^{-1}\right)\left(\left(y^{*} x\right)^{*}\left(y^{*} x\right)^{-1}\right)\left(y^{*} y^{-1}\right)$ and products of unitary elements are unitary, it will suffice to show $\left(d^{*} d^{-1}\right)\left(d^{*} d^{-1}\right)^{*} \equiv 1$ for any $d \in D^{\times}$. We have the same cases as in the proof of Lemma 2.10 (b). If $d+d^{*}=0$, then $d^{*} d^{-1}=-1$ and the conclusion follows. Otherwise, the result follows by applying part (d) to equation (2.11) since $d^{*} d^{-1} \in U$.

THEOREM 2.13. Let $v$ be the order valuation of $a^{*}$-semiordering $P$. Then $v$ is $a^{*}$-valuation.

ProOF. By [H2, Lemma 5.4], it will suffice to show that $\left[D^{\times}, D^{\times}\right] \subseteq A$. Let $x, y \in D^{\times}$. Then

$$
[x, y]=\left(\left(x^{*}\right)^{*}\left(x^{*}\right)^{-1}\right)\left(\left(y^{*} x\right)^{*}\left(y^{*} x\right)^{-1}\right)\left(y^{*} y^{-1}\right),
$$

a product of three units in $A$ by Lemma 2.10 (b). 
THEOREM 2.14. Let $v$ be the order valuation of $a^{*}$-semiordering $P$. Then $v$ collapses $\left[S(D)^{\times}, S(D)^{\times}\right]$. In particular, $v$ is a real valuation (cf. Proposition 2.3(b)).

PROOF. Let $x, y \in S(D)^{\times}$and write $[x, y]=s+k$, where $s^{*}=s, k^{*}=-k$. By Lemma 2.12(e), we have $1 \equiv[x, y][x, y]^{*} \equiv s^{2}-k^{2}$. If $s^{2} \equiv 1$, then $k \equiv 0$, so $[x, y] \equiv s \equiv \pm 1$. In this case, $2 s=\left([x, y]+[x, y]^{*}\right) \in P$, so $s \equiv 1$. Now assume $s^{2} \not \equiv 1$, so $k \not \equiv 0$. Since $s^{2} \equiv 1+k^{2}<1$, we obtain $s^{2} \leq q<1$ for some $q \in \mathbf{Q}^{+}$. But then $q-s^{2} \in P$ implies $q s^{2}-s^{4}=s\left(q-s^{2}\right) s \in P$, so that $s^{4} \leq q s^{2} \leq q^{2}$. By induction, we obtain $s^{2^{n}} \leq q^{2^{n-1}}$ for all positive integers $n$. On the other hand, set $[x, y]^{2^{n}}=s_{n}+k_{n}$, where $s_{n}^{*}=s_{n}, k_{n}^{*}=-k_{n}$. Then $0<s_{n+1}=s_{n}^{2}+k_{n}^{2} \leq s_{n}^{2}$, so we obtain $s_{n} \leq s^{2^{n}}$ for all $n$ by induction. Now $\left[x^{2^{n}}, y\right]=x^{2^{n-1}}\left[x^{2^{n-1}}, y\right] x^{-2^{n-1}}\left[x^{2^{n-1}}, y\right] \equiv\left[x^{2^{n-1}}, y\right]^{2}$ by Lemma 2.12(d), hence $\left[x^{2^{n}}, y\right] \equiv[x, y]^{2^{n}}$ by induction. Thus $s_{n}^{2}-k_{n}^{2} \equiv 1$ by Lemma 2.12(e). Also $0<$ $s_{n}^{2}+k_{n}^{2}$, so $2 \bar{s}_{n}^{2} \geq 1$ and $s_{n}^{2}>1 / 4$. Putting together our estimates, it follows that $q^{2^{n-1}} \geq s^{2^{n}} \geq s_{n}>1 / 2$ for all positive $n$, a contradiction. (The induction technique of this proof is due to Chacron [Ch, Theorem 1.5].)

In general, the valuation $v$ is not compatible with $P$. However, we can show compatibility if $v$ is discrete.

Lemma 2.15 (CF. [P2, Lemma 1.12]). Assume $x, y \in S(D)$ with $v(x)<$ $v(y), x>0$ and there exists $\gamma \in \Gamma$ such that $v(x) \leq 2 \gamma \leq v(y)$. Then $y<x$.

PROOF. Let $d \in D$ with $v(d)=\gamma$. Then $v(x) \leq v\left(d d^{*}\right) \leq v(y)$. If $v(x)<v\left(d d^{*}\right)$, then $d^{-1} x d^{-1 *} \notin A$ and $d^{-1} y d^{-1 *} \in A$. If $v\left(d d^{*}\right)<v(y)$, then $d^{-1} x d^{-1 *} \notin \mathfrak{m}$ and $d^{-1} y d^{-1 *} \in \mathfrak{m}$. In both cases we obtain $d^{-1} x d^{-1 *}>d^{-1} y d^{-1 *}$, and hence $x>y$.

When $v$ is discrete, the value group $\Gamma=\mathbf{Z}$ satisfies the hypothesis of the preceding lemma, so an immediate consequence is the following:

Proposition 2.16. If the order valuation of $a^{*}$-semiordering $P$ is discrete, then it is compatible with $P$.

As with Holland's strong orderings (herein called extended *-orderings), it is also possible to develop a theory of ${ }^{*}$-semiorderings which include nonsymmetric elements. This is indicated briefly in the remainder of this section.

DEFINITION 2.17. An extended ${ }^{*}$-semiordering is a ${ }^{*}$-closed subset $P \subseteq D^{\times}$ satisfying $P+P \subseteq P ; 1 \in P ; d P d^{*} \subseteq P$ for all $d \in D^{\times} ; P \cup-P \supseteq \Pi S(D)$; $P \cap-P=\varnothing$; and $P \cdot \Sigma \subseteq P$.

It follows immediately from the definition that every extended *-ordering is an extended *-semiordering. Furthermore, if $P$ is an extended *-semiordering, then $P \cap S(D)$ is a ${ }^{*}$-semiordering.

PROPOSITION 2.18. If $P$ is $a^{*}$-semiordering, then $P^{e}=\left\{\sum x_{i} \mid x_{i} \in \Pi S(D)\right.$. $\left.\Sigma, x_{i}+x_{i}^{*} \in P\right\}$ is an extended ${ }^{*}$-semiordering satisfying $P^{e} \cap S(D)=P$.

ProOF. The conditions in Definition 2.17 are easily checked for $P^{e}$ with the aid of the following lemma.

LEMMA 2.19. If $P$ is $a^{*}$-semiordering, then $p x+x^{*} p^{*}$ is contained in $P$ whenever $x \in \Sigma, p \in S(D) \cdot \Sigma$ and $p+p^{*} \in P$. 
ProOF. Write $p=s z$ with $s \in S(D)^{\times}, z \in \Sigma$. We must have $s \in P$, since otherwise, $s \in-P$ implies $p+p^{*}=s z+z^{*} s$ lies in $-P$. Now $p x+x^{*} p^{*}=$ $s(z x)+(z x)^{*} s \in P$ since $z x \in \Sigma$.

THEOREM 2.20. Any *-semiordering $P$ which is compatible with its order valuation $v$ is contained in an extended ${ }^{*}$-semiordering

$$
Q=\left\{s+k \mid s \in P, k^{*}=-k, v(k)>v(s)\right\} .
$$

Furthermore, $Q$ is again compatible with $v$.

PROOF. The proof can be carried out exactly as in [Cr, Theorem 2.5] if we have one additional fact: if $x \in \Sigma$ or $x \in \Pi S(D)$ with $x=s+k, s^{*}=s, k^{*}=-k$, then $v(k)>v(s)$. By Theorem 2.14, $v$ is a real ${ }^{*}$-valuation. By Theorem 3.4 (to be proved later), there exists $\mathrm{a}^{*}$-ordering, and hence an extended ${ }^{*}$-ordering $P^{e}$ compatible with $v$. Now either $x$ or $-x$ lies in $P^{e}$, so $v(k)>v(s)$ by the corresponding theorem for ${ }^{*}$-orderings [Cr, Theorem 2.5].

REMARK. Some ${ }^{*}$-semiorderings are not compatible with their order valuations, or indeed, with any real *-valuation. This happens even with $D$ commutative and ${ }^{*}=$ identity as shown in [P1, Theorem 7.17].

3. Lifting theorems. In $\S 4$ we make extensive use of theorems for lifting orderings from the residue ${ }^{*}$-field $D_{v}$. In general, this requires the existence of a collection of automorphisms of $D_{v}[\mathbf{H} \mathbf{1}, \mathbf{C r}]$. When the valuation collapses $\left[S(D)^{\times}, S(D)^{\times}\right]$, these automorphisms can all be chosen to be the identity and the theory becomes much simpler [Cr, Theorem 2.3]. In this section we shall first present simplified versions of the lifting theorems proved in $[\mathbf{C r}]$, but applied to ${ }^{*}$-semiorderings, and then prove a new theorem for real ${ }^{*}$-valuations which will also be needed later.

For any ${ }^{*}$-field $D$, we denote the set of all Baer orderings of $D$ by $Y_{D}$ and the subset of all *-semisuborderings by $Y_{D}^{*}$.

Let $v$ be a ${ }^{*}$-valuation on $\left(D,{ }^{*}\right)$ and assume $S(\Gamma)$ is a group. Define a mapping $s: S(\Gamma) \rightarrow S(D)^{\times}$as follows: $s(0)=1$; for each $\delta \in \Gamma$, choose $d \in D^{\times}$such that $v(d)=\delta$ and set $s(2 \delta)=d d^{*}$. Let $\left\{\beta_{i}\right\}$ be a set of representatives in $S(\Gamma)$ for a $\mathbf{Z} / 2 \mathbf{Z}$-basis of $S(\Gamma) / 2 \Gamma$, and for each $i$, choose any element $s\left(\beta_{i}\right) \in S(D)^{\times}$with $v\left(s\left(\beta_{i}\right)\right)=\beta_{i}$. Finally, any element of $S(\Gamma)$ has the form $\Sigma \beta_{i}+2 \delta$ and we define

$$
s\left(\Sigma \beta_{i}+2 \delta\right)=d\left(s\left(\beta_{1}\right) \cdots s\left(\beta_{n}\right)+s\left(\beta_{n}\right) \cdots s\left(\beta_{1}\right)\right) d^{*},
$$

where $s(2 \delta)=d d^{*}$. With this definition for $s$, we have (cf. [Cr, Theorem 2.7])

(1) for $\gamma \in S(\Gamma), s(\gamma+2 \delta)=d s(\gamma) d^{*}$ for some $d$ with $v(d)=\delta$; and

(2) for $\gamma_{1}, \gamma_{2} \in S(\Gamma)$, there exist $d \in U_{v}$ and $c \in\left[D^{\times}, S(D)^{\times}\right]$such that

$$
s\left(\gamma_{1}+\gamma_{2}\right)=d\left(\operatorname{cs}\left(\gamma_{1}\right) s\left(\gamma_{2}\right)+s\left(\gamma_{2}\right) s\left(\gamma_{1}\right) c^{*}\right) d^{*} \text {. }
$$

We shall call a mapping $s$ defined as above a semisection.

Proposition 3.1. Let $v: D^{\times} \rightarrow \Gamma$ be $a^{*}$-valuation collapsing $\left[S(D)^{\times}, S(D)^{\times}\right]$ with residue ${ }^{*}$-field $D_{v}$ and semisection $s$ as above. Then every ${ }^{*}$-semiordering $P$ of $D$ compatible with $v$ induces mappings

$$
\wp_{\Gamma}: S(\Gamma) / 2 \Gamma \rightarrow Y_{D_{v}}^{*} \quad \text { and } \quad \sigma_{P}: S(\Gamma) / 2 \Gamma \rightarrow\{ \pm 1\}
$$


defined by

(i) $\sigma_{P}(\bar{\gamma}) s(\gamma) \in P$ for all $\gamma \in S(\Gamma)$ and

(ii) for any $\bar{b} \in S\left(D_{v}\right)^{\times}, \bar{b} \in \wp_{P}(\bar{\gamma}) \Leftrightarrow$ for each lifting $\gamma$ of $\bar{\gamma}$, there exists $a \in P$ with $v(a)=\gamma$ and $\bar{b}=\overline{a s(\gamma)^{-1} \sigma_{P}(\bar{\gamma})}$.

PROOF. In [Cr, Lemma 3.1] this is proved for Baer orderings. The only additional thing to check is that $\wp_{P}(\bar{\gamma})$ is a ${ }^{*}$-semiordering when $P$ is. Let $\bar{p} \in \wp_{P}(\bar{\gamma})$ and $x \in \Sigma(D)$ with $v(x)=0$. Then there exists $a \in P$ with $v(a)=\gamma$ and $\bar{p}=\overline{a s(\gamma)^{-1} \sigma_{P}(\bar{\gamma})}$. Now $a x+x^{*} a \in P$, so

$$
\begin{aligned}
\bar{p} \bar{x}+\bar{x}^{*} \bar{p} & =\overline{a s(\gamma)^{-1} \sigma_{P}(\bar{\gamma}) x}+\overline{x^{*} a s(\gamma)^{-1} \sigma_{P}(\bar{\gamma})} \\
& =\overline{a x s(\gamma)^{-1} \sigma_{P}(\bar{\gamma})}+\overline{x^{*} a s(\gamma)^{-1} \sigma_{P}(\bar{\gamma})} \\
& =\overline{\left(a x+x^{*} a\right) s(\gamma)^{-1} \sigma_{P}(\bar{\gamma})}
\end{aligned}
$$

lies in $\wp_{P}(\bar{\gamma})$.

Proposition 3.2. Let $v: D^{\times} \rightarrow \Gamma$ be $a^{*}$-valuation collapsing $\left[S(D)^{\times}, S(D)^{\times}\right]$ with residue ${ }^{*}$-field $D_{v}$ and semisection $s: S(\Gamma) \rightarrow D^{\times}$. Consider any two functions $\wp: S(\Gamma) / 2 \Gamma \rightarrow Y_{D_{v}}^{*}$ and $\sigma: S(\Gamma) / 2 \Gamma \rightarrow\{ \pm 1\}$ such that $\sigma(\overline{0})=1$. These functions induce $a^{*}$-semiordering $P \in Y_{D}^{*}$ compatible with $v$ defined by

$$
a \in P \Leftrightarrow \overline{a s(v(a))^{-1} \sigma(\overline{v(a)})} \in \wp(\overline{v(a)}) .
$$

Proof. From [Cr, Lemma 3.2], we have everything except that the Baer ordering $P$ is a ${ }^{*}$-semiordering. Let $a \in P$ and $x \in \Sigma(D)$. Note that $v\left(a x+x^{*} a\right)=$ $v(a x)+v\left(1+x^{-1} a^{-1} x^{*} a\right)=v(a x)$ since $v$ collapses $\left[S(D)^{\times}, S(D)^{\times}\right]$, and hence also $\left[S(D)^{\times}, D^{\times}\right]$. Writing $\gamma$ for $v(a)$, we have $\overline{a s(\gamma)^{-1} \sigma(\bar{\gamma})} \in \wp(\bar{\gamma})$ and $\overline{x s(v(x))^{-1}} \in$ $\Sigma\left(D_{v}\right)$, the latter using the fact that $v(x)$ is even, so $s(v(x))$ is a norm. Thus $\wp(\bar{\gamma})$ contains

$$
\begin{gathered}
\overline{a s(\gamma)^{-1} \sigma(\bar{\gamma})} \overline{x s(v(x))^{-1}}+\overline{s(v(x))^{-1} x^{*}} \overline{a s(\gamma)^{-1} \sigma(\bar{\gamma})} \\
=\overline{\left(a x+x^{*} a\right) s(\gamma)^{-1} s(v(x))^{-1} \sigma(\bar{\gamma})} .
\end{gathered}
$$

Property (2) of the mapping $s$ implies that if we replace $s(\gamma)^{-1} s(v(x))^{-1}$ by $s(v(a x))^{-1}$, we remain in the ${ }^{*}$-semiordering $\wp(\bar{\gamma})$. Thus $a x+x^{*} a \in P$.

THEOREM 3.3 (CF. [Cr, THEOREM 3.4]). Let $v: D^{\times} \rightarrow \Gamma$ be a ${ }^{*}$-valuation collapsing $\left[S(D)^{\times}, S(D)^{\times}\right]$with semisection $s: S(\Gamma) \rightarrow D^{\times}$. Then the constructions of Propositions 3.1 and 3.2 yield an invertible one-to-one correspondence between $Y_{D}^{* v}$ and

$$
\left\{\wp \mid \wp: S(\Gamma) / 2 \Gamma \rightarrow Y_{D_{v}}^{*}\right\} \times\{\sigma \mid \sigma: S(\Gamma) / 2 \Gamma \rightarrow\{ \pm 1\}, \sigma(\overline{0})=1\}
$$

where $Y_{D}^{* v}$ denotes the set of all ${ }^{*}$-semiorderings compatible with $v$.

REMARK. Using the concept of extended *-semiordering, and in particular the extension $P^{e}$ of Proposition 2.18, condition (ii) in Proposition 3.1 can be restated to appear very similar to the commutative theory [P1, Lemma 7.5]:

(ii) for $b \in S(D) \cap U_{v}, \bar{b} \in \wp_{P}(\bar{\gamma}) \Leftrightarrow b s(\gamma) \sigma_{P}(\bar{\gamma}) \in P^{e}$.

Our next theorem shows that for real *-valuations, we can lift *-orderings without the "smoothness" conditions of $[\mathbf{C r}]$ or the strong commutator conditions of the previous theorem. 
THEOREM 3.4. Let $v$ be a real ${ }^{*}$-valuation on $\left(D,{ }^{*}\right)$ with semisection $s: S(\Gamma)$ $\rightarrow D^{\times}$.

(1) Let $P \in Y_{D}^{v}$. Then $\bar{P}=\left\{\bar{a} \in D_{v}^{\times} \mid a \in P, v(a)=0\right\}$ lies in $Y_{D_{v}}$ and $\sigma(\bar{\gamma}) s(\gamma) \in P$ for all $\gamma \in S(\Gamma)$ defines a function $\sigma: S(\Gamma) / 2 \Gamma \rightarrow\{ \pm 1\}$.

(2) Let $\bar{P} \in X_{D_{v}}$ and $\sigma \in \operatorname{Hom}(S(\Gamma) / 2 \Gamma,\{ \pm 1\})$. Then

$$
P=\left\{a \in S(D) \mid \overline{a s(v(a))^{-1} \sigma(\overline{v(a)})} \in \bar{P}^{e}\right\}
$$

lies in $X_{D}^{v}$.

(3) The processes of (1) and (2) give a bijective correspondence between $X_{D}^{v}$ and $X_{D_{v}} \times \operatorname{Hom}(S(\Gamma) / 2 \Gamma,\{ \pm 1\})$.

PROOF. (1) The properties of the mapping $s$ guarantee that $\sigma$ is well defined. Let $a, b \in U_{v} \cap S(D)$ with $b=a+m, m \in \mathfrak{m}$. Then $v(b)=0<v(m)$ and $P$ compatible with $v$ imply that $a=b-m \in P$. Thus the definition of $\bar{P}$ does not depend on the choice of representative. The axioms for a Baer ordering and the fact (for (3)) that $\bar{P} \in X_{D_{v}}$ when $P \in X_{D}^{v}$ can be easily checked.

(2) It is easily seen that $P \cap-P=\varnothing$ and $P \cup-P=S(D)^{\times}$. Let $a \in P$ and $d \in D^{\times}$. Then $v\left(d a d^{*}\right) \equiv v(a)(\bmod 2 \Gamma)$ and $s\left(v\left(d a d^{*}\right)\right)=c s(v(a)) c^{*}$ for some $c \in D^{\times}$with $v(c)=v(d)$ by property (1) of the mapping $s$. Therefore

$$
\left.\overline{d a d^{*} s\left(v\left(d a d^{*}\right)\right)^{-1} \sigma(\overline{v(a)})} \equiv \overline{c^{*-1} d} \overline{a s(v(a))^{-1} \sigma(\overline{v(a)})} \overline{\left(c^{*-1} d\right.}\right)^{*}
$$

$\left(\bmod \Sigma\left(D_{v}\right)\right)$, hence lies in $\bar{P}^{e}$, and so $d a d^{*} \in P$. Next, assume $a, b \in P$. If $v(a)=v(b)$, then

$$
\begin{aligned}
& \overline{(a+b) s(v(a+b))^{-1} \sigma(\overline{v(a+b)})} \\
& \quad=\overline{a s(v(a))^{-1} \sigma(\overline{v(a)})}+\overline{b s(v(b))^{-1} \sigma(\overline{v(b)})} \in \bar{P}^{e},
\end{aligned}
$$

so $a+b \in P$. Otherwise, we may assume $v(a)<v(b)$. Then $v(a \pm b)=v(a)$ and $b s(v(a))^{-1} \in \mathfrak{m}$, so we obtain $a \pm b \in P$. This gives closure under addition and compatibility of $P$ with $v$ since $0<a \leq b$ implies $a-b \notin P$, hence $v(b) \leq v(a)$. To check the multiplicative property, let $a_{1}, a_{2} \in P$, so

$$
\overline{a_{i} s\left(v\left(a_{i}\right)\right)^{-1} \sigma\left(\overline{v\left(a_{i}\right)}\right)} \in \bar{P}^{e} \quad \text { for } i=1,2 .
$$

Modulo $\Sigma\left(D_{v}\right)$, this gives

$$
\overline{a_{1} a_{2} s\left(v\left(a_{1}\right)\right)^{-1} s\left(v\left(a_{2}\right)\right)^{-1} \sigma\left(\overline{v\left(a_{1}\right)}\right) \sigma\left(\overline{v\left(a_{2}\right)}\right)}
$$

and

$$
\overline{a_{2} a_{1} s\left(v\left(a_{1}\right)\right)^{-1} s\left(v\left(a_{2}\right)\right)^{-1} \sigma\left(\overline{v\left(a_{1}\right)}\right) \sigma\left(\overline{v\left(a_{2}\right)}\right)}
$$

in $\bar{P}^{e}$. Adding these and using property (2) of the mapping $s$ and the fact that $\sigma$ is a homomorphism, we obtain

$$
\overline{\left(a_{1} a_{2}+a_{2} a_{1}\right) s\left(v\left(a_{1} a_{2}\right)\right)^{-1} \sigma\left(\overline{v\left(a_{1} a_{2}\right)}\right)} \in \bar{P}^{e},
$$

which shows that $a_{1} a_{2}+a_{2} a_{1} \in P$.

Finally, we must check that the processes in (1) and (2) are inverse to each other. Let $P \in X_{D}^{v}$ with $\bar{P}$ and $\sigma$ defined by (1) and $Q \in X_{D}$ obtained via (2) from $(\bar{P}, \sigma)$. Let $a \in Q$. Then $\overline{a s(v(a))^{-1} \sigma(\overline{v(a)})} \in \bar{P}^{e}$ by (2) and thus, by (1), we obtain an 
element $b \in P$ with $v(b)=0$ and $b=a s(v(a))^{-1} \sigma(\overline{v(a)})+m$ for some $m \in \mathfrak{m}$. Hence $a=b s(v(a)) \sigma(\overline{v(a)})+m s(v(a)) \sigma(\overline{v(a)})$, where the first term lies in $P^{e}$ since $s(v(a)) \sigma(\overline{v(a)}) \in P$ and the second term has larger value than the first. It follows by compatibility that $a \in P^{e} \cap S(D)=P$.

Conversely, it is easily checked that the other composition also gives the identity (cf. [Cr, Theorem 3.4; P, Theorem 7.8]).

4. Spaces of orderings. Let $\left(D,{ }^{*}\right)$ be a ${ }^{*}$-field. As in the previous sections, we use $X_{D}$ (resp. $Y_{D}$ ) to denote the set of all *-orderings (resp. Baer orderings) of $D$. The *-semiorderings $Y_{D}^{*}$ form a special subclass of $Y_{D}$ containing $X_{D}$. As in the commutative case with $^{*}=$ identity, we can topologize these sets to make them into Boolean spaces (compact, Hausdorff and totally disconnected).

For $d \in S(D)^{\times}$, define $H(d)=\left\{P \in Y_{D} \mid d \in P\right\}$. Note that $H(1)=Y_{D}$, $H(-1)=\varnothing$ and $H(-d)$ is the complement of $H(d)$. Let $\mathscr{H}=\left\{H(d) \mid d \in S(D)^{\times}\right\}$ be a subbasis for the topology on $Y_{D}$. The elements of $\mathscr{H}$ are clopen (i.e. both closed and open), so the space $Y_{D}$ is totally disconnected and Hausdorff; indeed, if $P, Q \in Y_{D}$, then there exists $d \in S(D)^{\times}$such that $d \in P, d \notin Q$, and therefore $Y_{D}=H(d) \cup H(-d)$ is a disconnection of the space which separates $P$ and $Q$.

A straightforward modification of the standard arguments (cf. [P1, Theorem 6.5]) shows that $Y_{D}$ is compact with $X_{D}$ and $Y_{D}^{*}$ closed subspaces, hence also compact with the induced topology. Henceforth, the notation $\mathscr{H}$ and $H(d)$ will refer to the space $X_{D}$, which will be the only one of these three spaces in which we will be working.

Among the most studied properties of $X_{F}$ for a commutative formally real field $F$ are the approximation properties, beginning with the work in [KRW and EL].

DEFINITION. A *-field $D$ is said to satisfy the strong approximation property (SAP) if $X_{D} \neq \varnothing$ and any two disjoint closed subsets $A$ and $B$ of $X_{D}$ can be separated by an element $d \in S(D)$; that is, $d \in P$ for all $P \in A$ and $-d \in P$ for all $P \in B$.

As in the commutative case, it is easy to see that the strong approximation property is equivalent to either of the following two conditions (see, for example, [P1, Proposition 6.6]):

(a) $\mathscr{H}$ is closed under finite intersections.

(b) $\mathscr{H}$ consists of all clopen subsets of $X_{D}$.

DEFINITION. A *-field $D$ is said to satisfy the weak approximation property (WAP) if $X_{D} \neq \varnothing$ and any point in $X_{D}$ can be separated from any closed subset of $X_{D}$ not containing the point by an element $d \in S(D)$.

This can easily be shown to be equivalent to $\mathscr{H}$ being a basis for the topology [P1, Proposition 6.6].

Many characterizations have been given for the (commutative) fields which satisfy SAP. Many of the most important characterizations can be found in $[\mathbf{P 1}$, Theorem 9.1], and it is basically that result which we shall now generalize to *-fields using the machinery set up in the previous sections.

THEOREM 4.1. Let $\left(D,{ }^{*}\right)$ be $a^{*}$-field with $0 \notin \Sigma(D)$. Then the following conditions are equivalent:

(a) $\left(D,{ }^{*}\right)$ satisfies $S A P$.

(b) $\left(D,{ }^{*}\right)$ satisfies WAP. 
(c) Every three nonarchimedean *-orderings of $D$ can be separated by some $d \in$ $S(D)$ from any other nonarchimedean *-ordering.

(d) For all real ${ }^{*}$-valuations $v$, we have $|S(\Gamma) / 2 \Gamma| \leq 2$ and if $|S(\Gamma) / 2 \Gamma|=2$, then $\left|X_{D_{v}}\right|=1$.

(e) Every ${ }^{*}$-semiordering is $a^{*}$-ordering.

(f) If $d_{1}, d_{2}, \ldots, d_{n} \in S(D)^{\times}$have the property that they never all have the same sign with respect to any ${ }^{*}$-ordering of $D$, then $0 \in \Sigma\left(\left\{d_{i}\right\}\right)$.

PROOF. (a) $\Rightarrow(\mathrm{b}) \Rightarrow(\mathrm{c})$ is trivial.

$(\mathrm{c}) \Rightarrow(\mathrm{d})$. Let $v$ be a real ${ }^{*}$-valuation and let $s$ be a semisection as defined in $\S 3$. Since $0 \notin \Sigma\left(D_{v}\right)$, there is a ${ }^{*}$-ordering $\bar{P}_{0} \in X_{D_{v}}$ [H2, Theorem 3.9].

Case 1: $|S(\Gamma) / 2 \Gamma| \geq 4$. Let $\left\{\bar{\gamma}_{i}\right\}_{i=1}^{m}, m \geq 2$, be a Z/2Z-basis for $S(\Gamma) / 2 \Gamma$. Define characters $\sigma_{i}: S(\Gamma) / 2 \Gamma \rightarrow\{ \pm 1\}$ by

$$
\begin{array}{lrrrr} 
& \sigma_{1} & \sigma_{2} & \sigma_{3} & \sigma_{4} \\
\bar{\gamma}_{1} & 1 & 1 & -1 & -1 \\
\bar{\gamma}_{2} & 1 & -1 & 1 & -1 \\
\bar{\gamma}_{k}, k>2 & 1 & 1 & 1 & 1
\end{array}
$$

By Theorem 3.4, there exist four ${ }^{*}$-orderings $P_{i}$ compatible with $v$ corresponding to $\left(\bar{P}_{0}, \sigma_{i}\right), i=1, \ldots, 4$. We note that each $P_{i}$ is nonarchimedean; indeed, choose any $a \in P_{i}$ with $v(a)<0$. Then, for any integer $n>0$, we have $v(n)=0$, $v(a)<v(n)$ and by compatibility, $a-n \in P_{i}$. We claim there is no $d \in S(D)$ with $P_{2}, P_{3}, P_{4} \in H(d), P_{1} \notin H(d)$. Write $\overline{v(d)}=n_{1} \bar{\gamma}_{1}+n_{2} \bar{\gamma}_{2}+\sum_{k>2} n_{k} \bar{\gamma}_{k}$. Then $\sigma_{i}(\overline{v(d)})=\sigma_{i}\left(\bar{\gamma}_{1}\right)^{n_{1}} \cdot \sigma_{i}\left(\bar{\gamma}_{2}\right)^{n_{2}}$. We have, by Theorem 3.4,

$$
d \in P_{i} \Leftrightarrow \overline{d s(v(d))^{-1} \sigma_{i}(\overline{v(d)})} \in \bar{P}_{0}^{e}
$$

Now this gives

$$
\begin{aligned}
& n_{1}=0, n_{2}=0: \quad d \in-P_{1} \cap P_{2} \Rightarrow \overline{d s(v(d))^{-1}} \in \bar{P}_{0}^{e} \cap-\bar{P}_{0}^{e}=\varnothing, \\
& n_{1}=0, n_{2}=1: \quad d \in-P_{1} \cap P_{3} \Rightarrow \overline{d s(v(d))^{-1}} \in \bar{P}_{0}^{e} \cap-\bar{P}_{0}^{e}=\varnothing, \\
& n_{1}=1, n_{2}=0: \quad d \in-P_{1} \cap P_{2} \Rightarrow \overline{d s(v(d))^{-1}} \in \bar{P}_{0}^{e} \cap-\bar{P}_{0}^{e}=\varnothing, \\
& n_{1}=1, n_{2}=1: \quad d \in-P_{1} \cap P_{4} \Rightarrow \overline{d s(v(d))^{-1}} \in \bar{P}_{0}^{e} \cap-\bar{P}_{0}^{e}=\varnothing \text {. }
\end{aligned}
$$

A contradiction in all cases implies that (c) fails to hold.

Case 2: $|S(\Gamma) / 2 \Gamma|=2$ and there exist two *-orderings $\bar{P}_{0} \neq \bar{P}_{1}$ in $X_{D_{v}}$. Let $\bar{\gamma}$ be a generator of $S(\Gamma) / 2 \Gamma$. Define characters $\sigma_{i}: S(\Gamma) / 2 \Gamma \rightarrow\{ \pm 1\}$ by $\sigma_{i}(\bar{\gamma})=(-1)^{i}$, $i=0,1$. By Theorem 3.4, we obtain four *-orderings in $X_{D}^{v}, P_{i, j}$, corresponding to $\left(\sigma_{i}, \bar{P}_{j}\right)$. Assume there exists an element $d \in S(D)$ such that $P_{0,0} \notin H(d)$ and $P_{1,0}, P_{0,1}, P_{1,1} \in H(d)$. Write $\overline{v(d)}=n \bar{\gamma}, n=0,1$. Then $\sigma_{i}(\overline{v(d)})=\sigma_{i}(\bar{\gamma})^{n}$ and $d \in P_{i, j} \Leftrightarrow \overline{d s(v(d))^{-1} \sigma_{i}(\bar{\gamma})} \in \bar{P}_{j}^{e}$. Again we obtain a contradiction in all cases:

$$
\begin{array}{ll}
n=0: & d \in-P_{0,0} \cap P_{1,0} \Rightarrow \overline{d s(v(d))^{-1}} \in \bar{P}_{0}^{e} \cap-\bar{P}_{0}^{e}=\varnothing, \\
n=1: & d \in P_{0,1} \cap P_{1,1} \Rightarrow \overline{d s(v(d))^{-1}} \in \bar{P}_{1}^{e} \cap-\bar{P}_{1}^{e}=\varnothing .
\end{array}
$$

$(\mathrm{d}) \Rightarrow(\mathrm{e})$. Let $P$ be a ${ }^{*}$-semiordering. Its order valuation $v$ is a real ${ }^{*}$-valuation by Theorems $2.13-2.14$. Since $|S(\Gamma) / 2 \Gamma| \leq 2$, the mapping $\sigma$ defined as in Theorem $3.4(1)$ is necessarily a homomorphism. The induced $\bar{P}$ on $D_{v}$ is a *-ordering since it is archimedean. 
Case 1: $|S(\Gamma) / 2 \Gamma|=1$. By Lemma 2.15, we have $P$ compatible with $v$. The conditions of [Cr, Theorem 4.3] are thus all met, showing that $P$ is a *-ordering.

Case 2: $|S(\Gamma) / 2 \Gamma|=2$. In this case we may not have $P$ compatible with $v$, so we define a new valuation $v_{1}$. Let $0<\gamma_{0} \in S(\Gamma), \gamma_{0} \notin 2 \Gamma$, and set $\Gamma^{\prime}=\{\gamma \in \Gamma \mid$ there does not exist $\gamma_{1} \in \Gamma$ with $\left.0<\gamma_{0}-2 \gamma_{1}<2|\gamma|\right\}$. Then $\Gamma^{\prime}$ is a convex subgroup of $\Gamma$ (cf. [P2]) so we obtain a new valuation $v_{1}: D \rightarrow \Gamma_{1}=\Gamma / \Gamma^{\prime}$, where the ordering of $\Gamma_{1}$ is defined by $\gamma_{1}+\Gamma^{\prime}<\gamma_{2}+\Gamma^{\prime}$ iff $\gamma_{1}<\gamma_{2}$ and $\gamma_{1}-\gamma_{2} \notin \Gamma^{\prime}$. We claim that $P$ is compatible with $v_{1}$; assume $x, y \in S(D)$ with $x>0$ and $v_{1}(x)<v_{1}(y)$. Then $v(x)<v(y)$ and we may assume $v(x)=\gamma_{0}, v(y)=\gamma_{0}+2 \gamma_{1}$ for some $\gamma_{1} \in \Gamma$. Now $v_{1}(x)<v_{1}(y)$ implies that $\gamma_{1} \notin \Gamma^{\prime}$, hence there exists $\gamma_{2} \in \Gamma$ such that $0<\gamma_{0}+2 \gamma_{2}<2 \gamma_{1}$. Therefore, $\gamma_{0}<2\left(\gamma_{0}+\gamma_{2}\right)<\gamma_{0}+2 \gamma_{1}$, so $y<x$ by Lemma 2.15 and we have compatibility of $v_{1}$ with $P$.

Write $D_{1}$ for the residue *-field of $v_{1}$. The value group of the place $D_{1} \rightarrow D_{v} \cup \infty$ is $\Gamma^{\prime}$ which has $\left|S\left(\Gamma^{\prime}\right) / 2 \Gamma^{\prime}\right|=1$. Thus the unique ${ }^{*}$-ordering of $D_{v}$ lifts uniquely to $D_{1}$ by Theorem 3.4. As in Case 1, we can now apply [Cr, Theorem 4.3] to $v_{1}$ and $D_{1}$, obtaining $P \in X_{D}$.

(e) $\Rightarrow$ (f). Assume that $0 \notin \Sigma\left(\left\{d_{i}\right\}\right)$. By Proposition 2.6, there exists a *semiordering $P$ containing either $\Sigma\left(\left\{d_{i}\right\}\right)$ or $\Sigma\left(\left\{-d_{i}\right\}\right)$. In fact $P$ is a ${ }^{*}$-ordering by (e). Thus the elements $d_{1}, \ldots, d_{n}$ all have the same sign with respect to a *-ordering.

(f) $\Rightarrow(\mathrm{a})$. Let $a, b \in S(D)^{\times}$. We shall show there exists an element $c \in S(D)$ with $H(a) \cap H(b)=H(c)$. By the multiplicative property of a *-ordering, the elements $1, a,-b$, and $a b+b a$ can never all have the same sign, and hence $0 \in$ $\Sigma(1, a,-b, a b+b a)$. Thus we may write $0=x_{1}+x_{1}^{*}+a x_{2}+x_{2}^{*} a-b x_{3}-x_{3}^{*} b+$ $(a b+b a) x_{4}+x_{4}^{*}(a b+b a)$, where each $x_{i} \in \Sigma \cup\{0\}$, with not all of them zero. Define $c:=-x_{1}-x_{1}^{*}+b x_{3}+x_{3}^{*} b=a x_{2}+x_{2}^{*} a+(a b+b a) x_{4}+x_{4}^{*}(a b+b a)$. Let $P \in X_{D}$.

Case 1: $c \neq 0$. If $a, b \in P$, then $c \in P$. If $b \in-P$, then $c \in-P$. If $b,-a \in P$, then $c \in-P$. Thus $H(a) \cap H(b)=H(c)$.

Case 2: $c=0$. Then $b x_{3}+x_{3}^{*} b=x_{1}+x_{1}^{*}$. If $x_{1}+x_{1}^{*} \neq 0$, then $b \in P$ for any ${ }^{*}$-ordering $P$, hence $H(b)=X_{D}$ and $H(a) \cap H(b)=H(a)$. If $x_{1}+x_{1}^{*}=0$, then $a x_{2}+x_{2}^{*} a=-(a b+b a) x_{4}-x_{4}^{*}(a b+b a) \neq 0$, so $a \in P$ implies $b \in-P$, and $a \in-P$ implies $b \in-P$; thus $H(b)=\varnothing$ and $H(a) \cap H(b)=H(-1)$.

Notice that this theorem leaves open the question of when the strong approximation property holds for the space of all Baer orderings $Y_{D}$, though it is solved for ${ }^{*}$-semiorderings as in the commutative case. We conclude this paper with some examples of *-fields satisfying SAP.

EXAMPLES 4.2. (i) The equivalent conditions of the theorem hold whenever $\left|X_{D}\right| \leq 3$ since, for topological reasons, closed sets can always be separated.

(ii) Part (c) of the theorem clearly applies to $\left(D,{ }^{*}\right)$ if all of its *-orderings are archimedean. In particular, this is true of any *-field algebraic over the rational numbers; i.e. any sub- ${ }^{*}$-field of the quaternions over the real algebraic numbers.

(iii) Let $R$ be a real closed field and let $D_{0}$ denote either $R$ or $C=R(i), i^{2}=-1$. Let ${ }^{*}$ denote the usual conjugation on $D_{0}\left({ }^{*}=\right.$ identity if $\left.D_{0}=R\right)$. Following [VG, pp. 35-36], we form $A=D_{0}\left[x,{ }^{*}\right]$, the skew polynomial ring in the variable $x$ with $x \alpha=\alpha^{*} x$ for $\alpha \in D_{0}$, and its field of fractions $D=D_{0}\left(x,{ }^{*}\right)$. The valuation rings in $D$, for valuations trivial on $R$, are $A_{(x)}, A_{\left(x^{-1}\right)}^{-1}$ (where $A^{-1}=D_{0}\left[x^{-1},{ }^{*}\right]$ ) and $A_{(p)}$ where $p \in R\left[x^{2}\right]$, the center of $A$, is irreducible in $A$. One easily checks that 
this means $p$ has the form $x^{2}+c$ with $c>0$ in $R$. We extend ${ }^{*}$ to $D$ as in $[\mathbf{C r}$, Example 5.1]; that is, $\left(\sum a_{k} x^{k}\right)^{*}=\sum_{\left(*^{r}\right)}(-1)^{k} a_{k}^{\left(*^{k+1}\right)} x^{k}$, where $a^{\left(*^{r}\right)}$ indicates that $a$ should be conjugated $r$ times; thus $a^{\left(*^{r}\right)}$ equals $a$ if $r$ is even and equals $a^{*}$ if $r$ is odd. In particular, $x=-x^{*}$ is a skew element.

All of the valuation rings above are in fact ${ }^{*}$-valuation rings (i.e. $d^{*} d^{-1}$ is in the ring for each $d \in D^{\times}$). The residue *-field is isomorphic to either $D_{0}$ (when localized at $(x)$ or $\left(x^{-1}\right)$ ) or, when localized at $p$, either $C$ or $H$ (the standard quaternion algebra over $R$ ) when $D_{0}=R$ or $C$, respectively. Each of these residue fields has a unique ${ }^{*}$-ordering. The value groups are all infinite cyclic and $S(\Gamma)=2 \Gamma$, so the valuation-theoretic condition of the theorem holds.

(iv) When $D_{0}=H$, the construction for $A$ above does not work. We consider insteaci $A=H[x]$, where $x$ is a central indeterminate. In this case, the valuation rings in $D$ containing $R$ are $A_{(x)}, A_{\left(x^{-1}\right)}^{-1}$ and $A_{(p)}$ where $p \in R[x]$, the center of $A$, and, being irreducible in $A$, is linear. If we define * to extend the usual conjugation on $D_{0}$ by $x^{*}=x$, then $S(D)=R(x)$ and one easily checks that the *-orderings of $D$ are in bijective correspondence with the usual orderings of $R(x)$ via restriction. In particular, $D$ is SAP since $R(x)$ is [EL, P1]. On the other hand, if we define the involution via $x^{*}=-x$ as in (iii), then $i x, j x \in S(D)$ with $[i x, j x]=-1$ so there are no ${ }^{*}$-orderings.

\section{REFERENCES}

[Ch] M. Chacron, $c$-orderable division rings with involution. II (to appear).

[Cr] T. Craven, Orderings and valuations on *-fields, Proc. Corvallis Conf. Quadratic Forms and Real Algebraic Geometry, Rocky Mountain J. Math. (to appear).

[EL] R. Elman and T.-Y. Lam, Quadratic forms over formally real fields and pythagorean fields, Amer. J. Math. 94 (1972), 1155-1194.

[H1] S. Holland, Jr., *-valuations and ordered *-fields, Trans. Amer. Math. Soc. 262 (1980), 219 243.

[H2] _ Strong orderings of *-fields, J. Algebra 101 (1986), 16-46.

[I] I. Idris, *-valuated division rings, orderings and elliptic hermitian spaces, Ph.D. thesis, Carleton University, Ottawa, Canada, 1986.

[K] M. Knebusch, Generalization of a theorem of Artin-Pfister to arbitrary semilocal rings, and related topics, J. Algebra 36 (1975), 46-67.

[KRW] M. Knebusch, A. Rosenberg and R. Ware, Signatures on semilocal rings, J. Algebra 26 (1973), 208 250.

[P1] A. Prestel, Lectures on formally real fields, Lecture Notes in Math., vol. 1093, Springer-Verlag, Berlin, 1984.

[P2] _ Q Quadratische Semi-Ordnungen und quadratische Formen, Math. Z. 133 (1973), 319342.

[S] O. Schilling, The theory of valuations, Math. Surveys, no. 4, Amer. Math. Soc., Providence, R.I., 1950.

[VG] J. Van Geel, Places and valuations in noncommutative ring theory, Lecture Notes in Pure and Appl. Math., no. 71, Marcel Dekker, New York, 1981.

Department of Mathematics, University of Hawail, Honolulu, Hawail 96822

Current address: Department of Mathematics, Oregon State University, Corvallis, Oregon 97331 